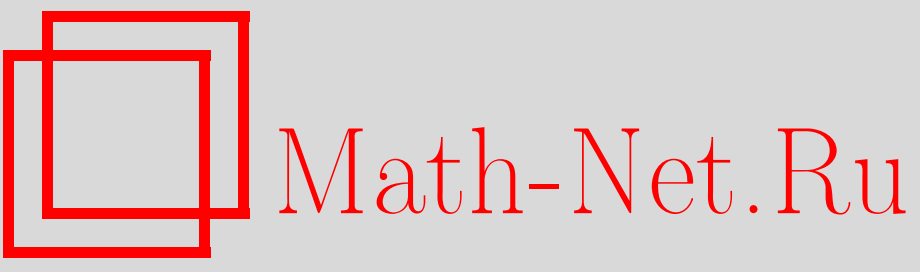

А. М. Райгородский, О хроматическом числе пространства, УМН, 2000, том 55, выпуск 2, 147-148

DOI: https://doi.org/10.4213/rm281

Использование Общероссийского математического портала Math-Net.Ru подразумевает, что вы прочитали и согласны с пользовательским соглашением

http://www . mathnet.ru/rus/agreement

Параметры загрузки:

IP : 34.229.108.108

26 апреля 2023 г., 15:47:16 


\section{О ХРОМАТИЧЕСКОМ ЧИСЛЕ ПРОСТРАНСТВА}

\section{А. М. РАЙГОРОДСКиЙ}

1. Введение и основной результат. Определим величину $\chi\left(\mathbb{R}^{n}\right)$ - хроматическое число евклидова пространства $\mathbb{R}^{n}$ - как минимальное число красок, необходимых для такой раскраски точек в $\mathbb{R}^{n}$, при которой между точками одного цвета никогда не реализуется евклидово расстояние, равное единице. Иными словами, $\chi\left(\mathbb{R}^{n}\right)$ - это хроматическое число следующего графа: множество вершин этого графра совпадает со всем $\mathbb{R}^{n}$, а ребрами соединены те и только те вершины, расстояние между которыми равно единице. Фактически, задача нахождения величины $\chi\left(\mathbb{R}^{n}\right)$ была впервые рассмотрена в работе [1] Хадвигером, который показал, что если имеется произвольное покрытие $\mathbb{R}^{n}=\bigcup_{i=1}^{n+1} \Omega_{i}$ замкнутыми множествами $\Omega_{i}$, то найдется такой элемент $\Omega_{i}$ этого покрытия, что $\mathbb{R}^{+} \cup\{0\}=\left\{|x-y|: x, y \in \Omega_{i}\right\}$. Известно (см. [2]-[4]), что $4 \leqslant \chi\left(\mathbb{R}^{2}\right) \leqslant 7$ и что, кроме того, $5 \leqslant \chi\left(\mathbb{R}^{3}\right) \leqslant 18$. В [5] Райский получил оценку $\chi\left(\mathbb{R}^{n}\right) \geqslant n+2$. Эта оценка неоднократно улучшалась: так, в [6] было доказано, что $\chi\left(\mathbb{R}^{n}\right) \gg n^{2}$, затем в [7] было получено неравенство $\chi\left(\mathbb{R}^{n}\right) \gg n^{3}$, и, наконец, в [8] была установлена экспоненциальность роста величины $\chi\left(\mathbb{R}^{n}\right)$. Иначе говоря, в работе [8] была доказана оценка $\chi\left(\mathbb{R}^{n}\right) \geqslant(1.207+o(1))^{n}$ (здесь следует отметить, что согласно [6] имеет место неравенство $\left.\chi\left(\mathbb{R}^{n}\right) \leqslant(3+o(1))^{n}\right)$. В настоящей работе с помощью техники, предложенной в [9], будет доказана следующая

Теорема. Положим $A_{1}=\frac{1}{2}(x+2 y), A_{2}=\sqrt{-3 A_{1}^{2}+6 A_{1}+1}, A_{3}=\frac{1}{4}\left(1+\frac{A_{1}-1}{A_{2}}\right) u$ $A_{4}=\frac{1}{6}\left(1+3 A_{1}-A_{2}\right)$ (здесь $x$ и $у$-независимые вещественные переменные). Определим, далее, величины $x_{0}, y_{0}$ как такие корни системь нелинейньх уравнений

(1)

$2 A_{3} \log A_{4}+\left(1-4 A_{3}\right) \log \left(A_{1}-2 A_{4}\right)+\left(2 A_{3}-1\right) \log \left(1-A_{1}+A_{4}\right)-\log y+\log (x-y)=0$,

$$
\frac{(1-x)^{2} y}{(x-y)^{3}}=1 \text {, }
$$

что $x_{0}=0.36063 \ldots$, а $y_{0}=0.063907 \ldots$ Наконец, зададим величину $\gamma$ равенством

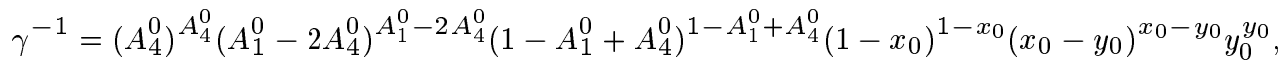

$$
\begin{aligned}
& \text { где } A_{i}^{0}=A_{i}\left(x_{0}, y_{0}\right) \text {. Тогда } \chi\left(\mathbb{R}^{n}\right) \geqslant(\gamma+o(1))^{n}=(1.239 \ldots+o(1))^{n} \text {. }
\end{aligned}
$$

2. Доказательство теоремы. Сперва заметим, что если $\Sigma \subset \mathbb{R}^{n}$ есть $(M, D)$-критическая конфигурация в $\mathbb{R}^{n}$ с критическим расстоянием $d>0$ (т.е. card $\Sigma=M$ и во всяком множестве $Q \subset \Sigma$ таком, что card $Q=D+1$, найдется пара элементов $x, y \in Q$, расстояние между которыми равно $d)$, то $\chi\left(\mathbb{R}^{n}\right) \geqslant M / D($ см. [6]), и, стало быть, для завершения доказательства теоремы нам достаточно построить некоторую $(M, D)$-критическую конфигурацию $\Sigma$ с $M / D \geqslant(\gamma+o(1))^{n}$.

Пусть $n \in \mathbb{N}$ достаточно велико, $a=\left[x_{0} n\right], b=\left[y_{0} n\right]$, а $p$ - наименьшее нечетное простое число, болшшее величины $[(a+2 b) / 2]$. Известно (см. [10; с. 364]), что для достаточно большого $x$ и для некоторого $\alpha<1$ (например, для $\alpha=38 / 61$ ) между $x$ и $x+x^{\alpha}$ лежит простое число. Поэтому можно считать, что $p<[(a+2 b) / 2]+[(a+2 b) / 2]^{\alpha}$. Заметим, что выбор величины $\alpha$ влияет исключительно на оценку величины $O(1)$ из формулировки теоремы. Положим $\Sigma=\left\{\mathbf{x}=\left(x_{1}, \ldots, x_{n}\right): x_{i} \in\{0,1,-1\} ; \operatorname{card}\left\{x_{i}= \pm 1\right\}=a ; \operatorname{card}\left\{x_{i}=-1\right\}=b\right\} \subset \mathbb{R}^{n}$. Понятно тогда, что $M=\operatorname{card} \Sigma=C_{n}^{a} C_{a}^{b}$. Ясно, кроме того, что conv $\Sigma$ есть кросс-политоп. В то же

Работа выполнена при финансовой поддержке Российского фонда фундаментальных исследований (гранты № № 99-01-00357, 00-15-96109). Автор благодарит професcopa László A. Székely за любезно предоставленный им еще неопубликованный обзор "Erdös on Unit Distances and the Szemerédi-Trotter Theorems". 
время в работе [8] оценки величины $\chi\left(\mathbb{R}^{n}\right)$ основывались на построении критических конфигураций, выпуклая оболочка которых представляет собой $(0,1)$-многогранник. Мы покажем здесь, что методы, предложенные в [11] и [9], позволяют работать с кросс-политопами и на их основе получать лучшие нижние оценки хроматического числа. В самом деле, как уже отмечалось выше, $M=C_{n}^{a} C_{a}^{b}$. Далее, нетрудно видеть, что имеет место следующее арифметическое свойство: для любых двух векторов $\mathbf{x}, \mathbf{y} \in \Sigma(\mathbf{x}, \mathbf{y}) \equiv a(\bmod p)$ в том и только том случае, когда либо $\mathbf{x}=\mathbf{y}$, либо $(\mathbf{x}, \mathbf{y})=a-p$.

Каждому вектору $\mathbf{x} \in \Sigma$ поставим в соответствие многочлен $F_{\mathbf{x}} \in \mathbb{Z} / p \mathbb{Z}\left[x_{1}, \ldots, x_{n}\right]$, полагая

$$
F_{\mathbf{x}}(\mathbf{y})=\prod_{i \neq \equiv a(\bmod p)}(i-(\mathbf{x}, \mathbf{y}))
$$

а затем, последовательно применяя соотношения $x_{i}^{3}=x_{i}, i=1, \ldots, n$, перейдем от многочлена $F_{\mathbf{x}}$ к многочлену $\widetilde{F}_{\mathbf{x}} \in \mathbb{Z} / p \mathbb{Z}\left[x_{1}, \ldots, x_{n}\right]$. Рассмотрим теперь произволное множество $Q=\left\{\mathbf{x}_{1}, \ldots, \mathbf{x}_{s}\right\} \subset \Sigma$ такое, что $\left(\mathbf{x}_{i}, \mathbf{x}_{j}\right) \not \equiv a(\bmod p) \forall i \neq j$. В этом случае можно показать, что многочлены $\widetilde{F}_{\mathbf{x}_{1}}, \ldots, \widetilde{F}_{\mathbf{x}_{s}}$ линейно независимы над полем $\mathbb{Z} / p \mathbb{Z}$ (см. [9]), а значит,

$$
s \leqslant \sum_{i=0}^{p-1} \sum_{j=0}^{[(p-1-i) / 2]} C_{n}^{j} C_{n-j}^{p-1-i-2 j}=D .
$$

Из неравенства (3) и арифметического свойства немедленно следует, что множество $\Sigma$ есть $(M, D)$-критическая конфигурация с критическим расстоянием $d=\sqrt{2 p}$ и, следовательно, $\chi\left(\mathbb{R}^{n}\right) \geqslant M / D$. Наконец, произведя несложные выкладки, нетрудно убедиться в том, что, в свою очередь, $M / D \geqslant(\gamma+o(1))^{n}$. Теорема доказана.

3. Замечания. 1. В доказательстве теоремы нет необходимости предполагать, что $p$-простое число. Достаточно считать, что $p=q^{\alpha}$, где $q$-простое, а $\alpha \geqslant 1$. При этом потребуется лишь внести некоторые изменения в определение многочленов $F_{\mathbf{x}}$ (см. [12]).

2. Хотя предложенный в настоящей работе метод получения нижних оценок хроматического числа пространства и дает асимптотически наилучший на данный момент результат, тем не менее в малых размерностях (по крайней мере при $n \leqslant 24$ ) улучшить с его помощью оценки из [5], [6] и [8] не удается. По-видимому, для получения дальнейших улучшений в рамках указанного метода необходимо существенно уточнить неравенство (3).

\section{СПИСОК ЛИТЕРАТУРЫ}

[1] Hadwiger H. // Portugal. Math. 1944. V. 4. P. 140-144. [2] Hadwiger H. // Elem. Math. 1961. V. 16. P. 103-104. [3] Moser L., Moser W. // Canad. Math. Bull. 1961. V. 4. P. 187-189. [4] Coulson D. // Discrete Math. 1997. V. 170. Р. 241-247. [5] Райский Д. Е. // Матем. заметки. 1970. Т. 7. C. 319-323. [6] Larman D. G., Rogers C. A. // Mathematika (London). 1972. V. 19. P. 1-24. [7] Larman D. G. // Comment. Math. Helv. 1978. V. 53. P. 529-535. [8] Frankl P., Wilson R. // Combinatorica. 1981. V. 1. P. 357-368. [9] Райгородский А. М. // УМН. 1999. Т. 54. № 2. С. 185-186. [10] Прахар К. Распределение простых чисел. М.: Мир, 1967. [11] Nilli А. // Contemp. Math. 1994. V. 178. Р. 209-210. [12] Райгородский А. М. // УМН. 1997. Т. 52. №6. С. 181-182.

Московский государственный

Принято редколлегией университет им. М. В. Ломоносова 26.01.2000 